\begin{tabular}{|c|c|c|c|}
\hline Eiszeitalter u. Gegenwart & $\mathbf{4 7}$ & $\begin{array}{c}28-41 \\
\text { 6 Abb., 1. Tab. }\end{array}$ & Hannover 1997 \\
\hline
\end{tabular}

\title{
Holozäne Einwanderungsgeschichte der Baumgattungen Picea und Quercus unter paläoökologischen Aspekten nach Europa
}

\author{
Anne Kathrin GLIEMEROTH*)
}

\author{
Holocene, tree taxa, immigration, first traces, mass \\ spreading, palaeoecological approach, overlayering maps, Picea, Quercus, Europe
}

\begin{abstract}
Kurzfassung: Das Ziel der Arbeit war es, für die Baumgattungen Picea und Quercus deren Einwanderungsgeschichte nach der Letzten Eiszeit aus ihren eiszeitlichen Refugien für Europa zu fassen. Um die Einwanderungsbewegungen besser darstellen zu können, wurden nicht Isopollenkarten verwendet, sondern eine Darstellung des Auftretens der 1. Spuren sowie der Massenausbreitung in Überlagerungskarten.

Die Zeitangaben für die Wanderbewegungen wurden nicht in ${ }^{14} \mathrm{C}$-Altern angeben, sondern in calibrierten Werten mit Hilfe von Jahrringen von Bäumen und U/Th-Daten von Korallen. Die Einwanderungskarten überdecken einen Zeitraum von $14^{\prime} 000 \mathrm{cal} .{ }^{14} \mathrm{C}$ yr. b. p. bis heute, wobei die einzelnen Baumgattungen verschiedene Zeitscheiben für ihre Wanderbewegungen, und zwar sowohl der 1. Spuren wie der Massenausbreitung, umfassen.

Die hier gewählte Darstellungsform der Einwanderungsbewegung in Überlagerungskarten für die ersten Spuren und die Massenausbreitung der Baumgattungen ermöglicht es, zeitlich-räumliche Aussagen über die Einwanderungsrichtung, die Einwanderungsbahnen, die maximal eingenommene Arealgrenze, die Einwanderungszeiten und die Einwanderungsgeschwindigkeit im Hinblick auf eine paläoökologische Interpretation dieser Prozesse zu machen.
\end{abstract}

\section{[Holocene immigration history of Picea and Quercus in Europe with special regard to palaeoecological aspects \\ mass budget of glaciers, Iller glaciers, Island]}

\begin{abstract}
The paper aims at studying the immigration history of Picea and Quercus since the Last Glaciation in Europe. The data are presented in maps showing the geographical distribution patterns of the first traces of the relevant taxa which point to the very beginning of immigration of the various taxa into the sites studied, not by isopollenmaps, but by overlayering-maps. Another set of maps informs about the geographical distribution patterns of the various taxa becoming important components of the vegetation.

The ages are given in calibrated ${ }^{14} \mathrm{C}$-years. The immigration maps cover the time from $14^{\prime} 000 \mathrm{cal} .{ }^{14} \mathrm{C}$ yr. b. p. till today. It becomes evident that there existed strong differences in time and in space regarding the immigration processes of the taxa studied.
\end{abstract}

*) Anschrift der Autorin: Dr. A. K. Gliemeroth, Institut für Botanik -210-, Universität Hohenheim, Garbenstr. 30, 70593 Stuttgart, Deutschland
The chosen form of visualizing the migration pattern of the taxa studied enabled a discussion based on regional and spatial aspects like direction, ways, time and velocity of immigration, with regard to a palaeoecological interpretation of these processes.

\section{Einleitung}

Schon früh wurde versucht, das Auftreten verschiedener Pollentypen in Kartenform darzustellen, da sich in einem Pollendiagramm die zeitliche Veränderung der Zusammensetzung des sedimentierten Pollens an einer beprobten Lokalität widerspiegelt und dies eine vorsichtige Ermittlung der zeitlichen Abfolge im Auftreten verschiedener Pflanzen und damit auch der Vegetationsänderungen ermöglicht. So zeichnete bereits 1935 SzAFER Einwanderungskarten von Fagus und Picea in Polen. Firbas erstellte 1949 Isopollenkarten für verschiedene Gehölzpflanzen in Zentral-Europa. In diesen Arbeiten mußte allerdings die Synchronisation der Pollendiagramme noch anhand der biostratigraphischen Pollenzonen und einer dadurch etablierten relativen Zeitskala durchgeführt werden.

Durch die Entwicklung der ${ }^{14} \mathrm{C}$-Methode zur Datierung organischen Materials ergab sich besonders für den Zeitraum des Spät- und Postglazials eine gute Datierungsmöglichkeit der Pollendiagramme. Diese Datierungen erlaubten es, zeitgleiche Schichten verschiedener Lokalitäten, unabhängig von ihrer Biostratigraphie, miteinander zu verknüpfen und somit ein größeres geographisches Gebiet zu bearbeiten. Diese nun erstellten sogenannten isochronen Pollenkarten konnten, neben der bereits guten räumlichen Auflösung auch eine gute zeitliche erreichen und wurden in den folgenden Jahren nicht nur regional angewandt (Fennoskandien: Donner, 1963; Aario, 1965; Aartholathi, 1966, 1967; MoE, 1970; Rußland: SEREBryanny, 1973; Britische Inseln: Birks et al., 1975), sondern auch großräumig (Nordamerika: Moran, 1973; Davis, 1976; Bernabo \& Webb, 1977; Europa: Huntley \& Birks, 1983). Die Problematik, daß die für ganz Europa erstellten Karten 
(HuNTLEY \& BiRKs, 1983) erheblich von denen der regionalen Karten abwichen (RALSKA-JASIEWICZOWA, 1983; RYBNICKOva \& RYBNICEK, 1988), ließ es notwendig erscheinen, das Thema erneut zu bearbeiten, verbunden mit einem möglichst detaillierten Literaturstudium.

Es kommt hinzu, daß inzwischen die Datierung mittels der ${ }^{14} \mathrm{C}$-Methode anhand der Dendrochronologie weiterhin kritisch überprüft worden war (STUIVER et al., 1986; 1993), so daß es nun möglich war, calibrierte Altersangaben zu erhalten, die dem tatsächlichen absoluten Alter sehr nahe zu kommen scheinen. In der vorliegenden Arbeit wurde diese Weiterentwicklung der Datierungsmethodik angewandt und die vorliegenden Karten wurden in calibrierten Jahren erstellt.

Einwanderungskarten einer Baumgattung können so entworfen werden, daß für mehr oder weniger regelmäßig aufeinanderfolgende Zeiten die jeweils erreichten Pollenprozentwerte der Baumgattung kartiert und durch Isolinien verbunden werden (,Isopollenkarten"), doch erschien es unter der paläoökologischen Fragestellung der Wanderbewegung der Baumgattungen im vorliegenden Fall von größerem Interesse, zwei Grenzsituationen in der Verbreitung einer Baumgattung aufzuzeigen, zum einen den Zeitpunkt der ersten Spuren der Baumgattung am Standort, zum anderen ihre Massenausbreitung. Zur Darstellung wurde die Form der „Überlagerungskarten“ gewählt, deren Vorteil gegenüber den eher quantitave Änderungen aufzeigenden „Isopollenkarten" in einem relativ schnellen Überblick über die Einwanderungsrichtung, die benutzten Einwanderungsbahnen, die maximal eingenommenen Arealgrenzen, die Einwanderungszeiten und die Einwanderungsgeschwindigkeit liegt.

Durch die Einwanderungskarten für die beiden Baumgattungen Picea und Quercus nach Europa im Verlaufe des Spät- und Postglazials sollte geprüft werden, wie sich die einzelnen Gehölzpflanzen bezüglich der Lage ihrer eiszeitlichen Refugien, ihrer Einwanderungsbahnen und ihrer Einwanderungszeiten sowie der Dauer des Einwanderungsprozesses verhalten haben. Denn es ist zu erwarten, daß solche Gehölzpflanzen, die sich in der Vergangenheit unterschiedlich verhalten haben, auch heute deutliche Unterschiede in ihrer Reaktion auf Veränderungen im Ökosystem zeigen werden.

Eine Frage, die sich bei der Wanderbewegung der Gehölzpflanzen aus ihren eiszeitlichen Refugien nach Europa stellt, ist die nach der Beeinflussung dieses Prozesses durch das Klima. Es erscheint äußerst wichtig, im Zusammenhang mit der Frage nach dem Einfluß des Klimas auf die Wanderbewegung der Gehölzpflanzen, zu beachten, daß es sich hierbei um einzelne Vegetations- oder auch Ökosystemtypen handelt, die als solche mehr oder weni- ger stark internen Regelmechanismen unterworfen sind (Frenzel, 1993b) und, daß ab dem Neolithikum mit einem Einfluß des Menschen auf die Wanderbewegungen der Gehölzpflanzen, besonders jener, die als Nutzbäume für den Menschen von Bedeutung waren, zu rechnen sein muß (Frenzel, 1992).

\section{Material und Methoden}

Aus der umfangreichen Datenbasis von 1700 Pollendiagrammen (GLIEMEROTH, 1995) wurden alle Pollendiagramme mit 5 oder mehr ${ }^{14} \mathrm{C}$-Datierungen ausgewählt. Dies erschien als Grundstock an Pollendiagrammen für die vorgesehene Calibrierung der ${ }^{14} \mathrm{C}$ Datierungen als realistisch, um eine möglichst genaue Aussage über das absolute Alter der Einwanderungsbewegungen der Gehölze zu machen.

Für die Einwanderungskarten der Gehölzpflanzen war es allerdings neben dieser guten Datierung notwendig, daß eine flächenhafte Überdeckung des europäischen Kontinents gegeben war. Daher wurden z. T. auch solche Pollendiagramme, die weniger oder keine ${ }^{14} \mathrm{C}-$ Daten hatten, aber als repräsentativ für einzelne Regionen gelten konnten, genutzt, soweit sie in ihrem Kurvenverlauf gut datierten Pollendiagrammen der Nachbarschaft entsprachen, um auch für solche Gebiete, die zwar gute pollenanalytische Arbeiten aufweisen, aber keine ${ }^{14} \mathrm{C}-\mathrm{Datierun}-$ gen, die Einwanderungsbewegung der Gehölzpflanzen fassen zu können.

In den Jahrringchronologien von Quercus und $\mathrm{Pi}$ nus kann in Europa der Unterschied zwischen ${ }^{14} \mathrm{C}-$ Alter und wahrem Alter ermittelt werden. Hierdurch konnte gezeigt werden, daß der langfristige Trend des ${ }^{14} \mathrm{C}$-Gehalts, der in der Regel der Veränderung im magnetischen Dipolmoment der Erde zugeschrieben wird, überlagert wird von schnellen Variationen im ${ }^{14} \mathrm{C}$-Gehalt von bis zu 500 Jahren (STUIVER et al., 1991). Durch diese Anomalien der ${ }^{14}{ }^{14} \mathrm{C}-\mathrm{Plateaus}$ " treten Fälschungen in der Radiokarbon-Zeitskala auf, die sich besonders dann bemerkbar machen, wenn es um das absolute Alter eines Ereignisses geht oder wenn die Dauer eines Ereignisses betrachtet werden soll, bzw. dann, wenn paläoökologische Analysen durchzuführen sind (Frenzel, 1975; BeCKer \& KroMER, 1993).

Da jedoch die Jahrringchronologie nur bis 11500 cal.Jahre zurückreicht, sind für die älteren Zeiten die ${ }^{230} \mathrm{Th} /{ }^{234} \mathrm{U}$-Datierungen an Korallen von Barbados, Galapagos und Mururoa als Calibrierungsgrundlage der ${ }^{14} \mathrm{C}-$ Datierungen von größter Bedeutung (BARD et al., 1993). Diese beiden Calibrierungsmöglichkeiten, die Dendrochronologie sowie die U/Th-Datierungen ermöglichen es, das konventionelle ${ }^{14} \mathrm{C}$-Alter in das höchstwahrscheinlich absolute Alter, angegeben in calibrierten Jahren vor heute, umzurechnen. In den meisten Fällen ist die Pollenanalyse nur in der 
Lage, eine Bestimmung bis zum Niveau der Gattung durchzuführen, so daß z. B. unter dem Begriff Picea 2 Arten dieser Gattung im Untersuchungsgebiet von Europa bis zum Ural enthalten sind (P.abies, P.omorika), bei Quercus allerdings 16 (Q. brachyphylla, Q. canariensis, $Q$. cerris, $Q$. congesta, $Q$ faginea, Q. fruticosa, Q. ilex, Q. infectoria, Q. macrolepis, Q. petraea, Q. pubescens, Q. pyrenaica, Q. robur, Q. sicula, Q. suber, Q. trojana). Hierbei ist es allerdings von Vorteil, daß es sich zum Teil um endemische oder recht selten auftretende Arten handelt.

In einer Tabelle soll versucht werden, für die wichtigsten Vertreter der Gattungen die Faktoren darzustellen, die für ihre Einwanderungsgeschichte von Bedeutung sind. Dies sind zum einen die klimatischen und die Lichtansprüche der Pflanzen, entweder als Baum oder als Keimling, zum anderen aber auch ihre Bedeutung für die Nutzung durch den Menschen und ihre Bestäubungsart (Tab. 1). Gleichzeitig sind auch diejenigen Pollenprozentwerte angegeben, die jeweils für die Konstruktion der Karten der „ersten Spuren des Vorkommens", bzw. der „Massenausbreitung" verwandt worden sind.

Die Festlegung der Pollenprozentwerte, die zur Beschreibung der 1. Spuren einer Baumgattung benutzt werden können, ist äußerst schwierig. Theoretisch gibt es 3 Möglichkeiten, die 1. Spuren einer Baumgattung am Standort zu definieren: 1. Es könnte darunter das erste Auftreten des Pollens im Pollendiagramm verstanden werden. Dies würde jedoch eine recht große Fehlerbreite beinhalten, da bei anemogamen Pflanzen ein sehr weiter Ferntransport über mehr als $100 \mathrm{~km}$ Distanz vorliegen kann und selbst der Pollen entomogamer Pflanzen über riesige Entfernungen verbreitet werden kann (vgl. Srodon, 1960; Frenzel, 1957), so daß die Aussagekraft des ersten Auftretens des Pollens im Pollendiagramm über die tatsächliche Anwesenheit der Baumgattung am Standort sehr gering sein kann. 2. Die ersten Spuren könnten aber auch mit dem Beginn der geschlossenen Pollenprozentkurve, der sogenannten empirischen Pollengrenze nach FirBAS (1949), definiert werden oder 3. aber mit dem Anstieg der Pollenprozentkurve über einen bestimmten Grenzwert, der sogenannten rationellen Pollengrenze nach FirBas (1949).

In der vorliegenden Arbeit wurden die ersten Spuren des Auftretens einer Baumgattung durch das Überschreiten eines bestimmten Pollenprozentwertes definiert. Dieser wurde für die einzelnen Baumgattungen anhand der Literatur festgelegt. Hierbei kann auf eine ungewöhnlich reichhaltige Literatur zurückgegriffen werden, die sich mit der pollenanalytischen Repräsentanz der einzelnen Sippen in Oberflächenproben, im Vergleich zur umgebenden Vegetation, auseinandersetzt (Literaturübersicht siehe Gliemeroth, 1995).

Neben dem Zeitpunkt der ersten Spuren des Auftretens einer Baumgattung wurde der Zeitpunkt der Massenausbreitung erfaßt, der Zeitpunkt also, an dem die Baumgattung am Standort relativ bedeutungsvoll wurde. Dies spiegelt nicht Dominanz über die anderen am Standort vorkommenden Bäume, sondern ebenfalls, wie beim Erfassen der ersten Spuren einer Baumgattung, das Überschreiten eines baumartspezifischen Pollenprozentwertes wider. Um durch die Auswahl der beiden Eckdaten, 1. Spuren und Massenausbreitung in der Einwanderungsgeschichte der Gehölzpflanzen Aussagen über den zeitlich-räumlichen Verlauf der Einwanderung der

Tab. 1: Faktoren, die für die wichtigsten Arten der untersuchten Gattungen, hinsichtlich der Einwanderung von Bedeutung sind (1. Sp. = 1. Spuren; MA = Massenausbreitung)

Table 1: Important factors for the spreading of the main species of the investigated tree genera

\begin{tabular}{|c|c|c|c|c|c|c|c|}
\hline Gattung & $\begin{array}{l}\text { Wichtige } \\
\text { Arten }\end{array}$ & $\begin{array}{l}\text { Klima- } \\
\text { Ansprüche }\end{array}$ & $\begin{array}{l}\text { Licht- } \\
\text { Ansprüche }\end{array}$ & $\begin{array}{l}\text { Menschliche } \\
\text { Nutzung }\end{array}$ & Verbreitung & $\begin{array}{l}\text { 1. Sp } \\
{[\%]}\end{array}$ & $\begin{array}{l}\text { MA } \\
{[\%]}\end{array}$ \\
\hline Picea & abies & frosthart & $\begin{array}{l}\text { Lichtkeimer, } \\
\text { Halbschattholz }\end{array}$ & Bauholz & Wind & $>2$ & $>7$ \\
\hline \multirow[t]{3}{*}{ Quercus } & robur & $\begin{array}{l}\text { sommerwarm } \\
\text { subkontinental }\end{array}$ & $\begin{array}{l}\text { Halblicht- } \\
\text { Halbschattholz }\end{array}$ & $\begin{array}{l}\text { Bauholz } \\
\text { Eichelmast, } \\
\text { Gerbrinde }\end{array}$ & Wind & $>2$ & $>7$ \\
\hline & petraea & $\begin{array}{l}\text { spätfrost- } \\
\text { empfindlich }\end{array}$ & $\begin{array}{l}\text { Halblicht- } \\
\text { Halbschattholz }\end{array}$ & $\begin{array}{l}\text { Nutzholz } \\
\text { Eichelmast, } \\
\text { Gerbrinde }\end{array}$ & Wind & $>2$ & $>7$ \\
\hline & pubescens & $\begin{array}{l}\text { wärmeliebend, } \\
\text { submediterran }\end{array}$ & $\begin{array}{l}\text { Halblicht- } \\
\text { Halbschattholz }\end{array}$ & $\begin{array}{l}\text { Eichelmast } \\
\text { Gerbrinde }\end{array}$ & Wind & $>2$ & $>7$ \\
\hline
\end{tabular}


einzelnen Gattungen und über die Zeitdauer vom Auftreten der ersten Spuren bis zur Massenausbreitung machen zu können, die sich anhand von „Isopollenkarten" nicht machen lassen, wurde die bei diesem Ansatz methodisch verursachte gewisse Fehlerbreite bei der Grenzziehung in Kauf genommen, da zum Beispiel das Areal der 1. Spuren des Pollens einer Baumgattung nicht immer unbedingt gleich der ehemaligen Verbreitungsgrenze sein muß. Auch war es anhand der „Überlagerungskarten“ möglich, die Zeitdauer zwischen den ersten Spuren und der Massenausbreitung einer Baumgattung innerhalb der verschiedenen Gattungen gebietsweise zu vergleichen, um aufzuzeigen, welche Baumgattung schnell an einem Standort von den ersten Spuren zur Massenausbreitung kommt und welche, bedingt durch ökologische Faktoren, länger dafür braucht. Für die Baumgattungen wurden die Einwanderungskarten ihrer ersten Spuren und ihrer Massenausbreitung gezeichnet. Hierbei wurde versucht, die Schwarz-Weiß-Schraffur derart zu gestalten, daß die dunkleren Flächen die älteren Zeiten wiedergeben. Die Angabe der Jahre ist, wie bereits erwähnt, in calibrierten Jahrtausenden vor heute [cal. ${ }^{14} \mathrm{C}$ yr b. p.] gemacht, die jeweilige Fläche stellt das Gebiet dar, in welches die untersuchte Baumgattung während 1000 calibrierter Jahre eingewandert war. Die Fläche „11" gibt also die Fläche an, die zwischen 11'999 und $11^{\prime} 000$ cal. Jahren vor heute von der Baumgattung besiedelt wurde. Die älteste Fläche beim Auftreten einer Baumgattung ist immer durch ein ," "Zeichen markiert, dies bedeutet, daß es durch die geringe Dichte von Pollendiagrammen, besonders in den eiszeitlichen Refugien der Baumgattungen, nicht ratsam erschien, eine Wanderbewegung der Baumgattung vor diesem Zeitpunkt zu fassen und die markierte Fläche nur das Gebiet kennzeichnet, in dem die Baumgattung bis zu diesem Zeitpunkt der Grenzziehung vorhanden war.

\section{Ergebnisse}

\subsection{Die Einwanderung der Baumgattungen Erste Spuren und Massenausbreitung}

\subsubsection{Picea (Fichte)}

Bei Picea liegt der ungewöhnliche Fall vor, daß neben den südosteuropäischen eiszeitlichen Refugien noch ein Refugium während der Letzten Eiszeit im Südural, vielleicht auch auf den Mittelrussischen Höhen bestanden hatte, aus dem Picea allerdings erst später mit einer Einwanderung nach Europa begonnen hat als aus dem südosteuropäischen Refugium (Abb. 1). Der Nachweis des Refugiums von Picea im Südural und auf den Mittelrussischen Höhen ist jedoch mit dem methodischen Problem der „unteren Fichte" der westlichen mittelrussischen
Höhen und der im Westen angrenzenden Landschaften eng verknüpft. Picea sowie thermophile Pflanzen treten nur im minerogenen Sediment auf, während der Pollennachweis sofort aufhört, wenn der Torf beginnt. Dies wirft die berechtigte Frage auf, ob Picea während des Alleröds tatsächlich dort vorhanden war, oder ob es sich nur um umgelagerten älteren Pollen handelt (Frenzel, 1960), so daß das Vorhandensein dieses Refugiums von Picea während der Letzten Eiszeit fraglich ist. Im Bereich, in dem die beiden Wanderbewegungen aus den eiszeitlichen Refugien aufeinandertreffen müßten, läßt sich dies erst ab $11^{\prime} 000$ bis $10^{\prime} 000$ cal. ${ }^{14} \mathrm{C}$ yr. b. p. in Ostpolen fassen, da in diesem Gebiet die Dichte der Pollendiagramme gegenüber dem vorher durchwanderten Rußland hinreichend ist, um eine Wanderbewegung von Picea zu fassen. Der russische Bereich der Konfluenz beider Einwanderungsrichtungen ist daher mit einem Fragezeichen gekennzeichnet. Die Einwanderungsbewegung verläuft hauptsächlich Ost-West, ist aber durch häufige regionale Stops gekennzeichnet.

Die Fichte beginnt mit ihrer Einwanderung aus den südosteuropäischen Refugien vor $14^{\prime} 000 \mathrm{cal} .{ }^{14} \mathrm{C}$ yr. b. p., erreicht bis $9^{\prime} 000$ cal. ${ }^{14} \mathrm{C}$ yr. b.p. eine Linie Poebene - Donauoberlauf - Mittelpolen - Luga-Mündung, stößt aber in den anschließenden 9000 cal. Jahren in Mitteleuropa nur noch in die Westalpen, bis an den Rhein und in das norddeutsche Flachland vor, während im gleichen Zeitraum Skandinavien besiedelt wird, wobei sich besonders in Schweden die von Norden bzw. Nordosten einsetzende Besiedlung von der anderer Baumgattungen unterscheidet. Picea ist eine der wenigen Holzgattungen, die heute anscheinend immer noch in Ausbreitung begriffen ist. Daß dies durch den menschlichen Einfluß, der zu Anpflanzungen von Picea außerhalb ihrer westlichen natürlichen Verbreitungsgrenze geführt hat, überlagert wird, ist bei der Nutzung des Picea-Holzes als Bauholz und von Inhaltsstoffen der Rinde als Gerbstoffe verständlich.

Die Massenausbreitung von Picea beginnt in Südosteuropa vor $10^{\prime} 000 \mathrm{cal} .{ }^{14} \mathrm{C}$ yr. b. p. und setzt sich dann bis $1^{\prime} 000 \mathrm{cal} .{ }^{14} \mathrm{C}$ yr. b. p. in Mitteleuropa nach Westen fort (Abb. 2). Ausgehend vom zweiten eiszeitlichen Refugium auf den Mittelrussischen Höhen oder im Südural, läßt sich eine Massenausbreitung in der Zeitscheibe 6'000 fassen, die sich in westlicher Richtung nach Skandinavien fortsetzt. Zwischen diesen beiden Gebieten, in denen es zur Massenausbreitung kommt, bleibt ein Gürtel von Norddeutschland, Südschweden, Nordpolen nach Osten, in dem Picea nicht zur Massenausbreitung gelangt.

\subsubsection{Quercus (Eiche)}

Während der Stadiale der Letzten Eiszeit hielt sich Quercus im gesamten Mittelmeergebiet in den dort 


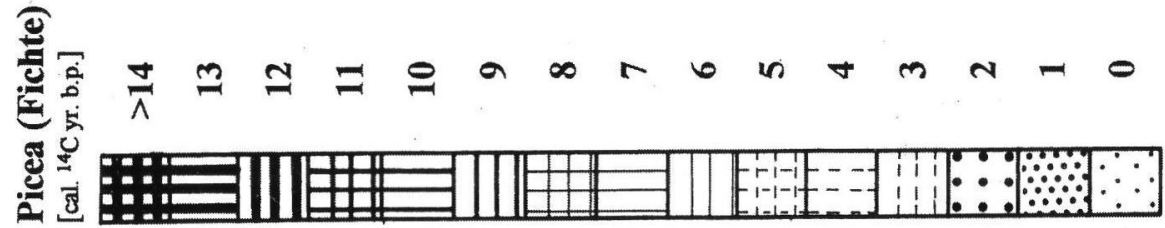

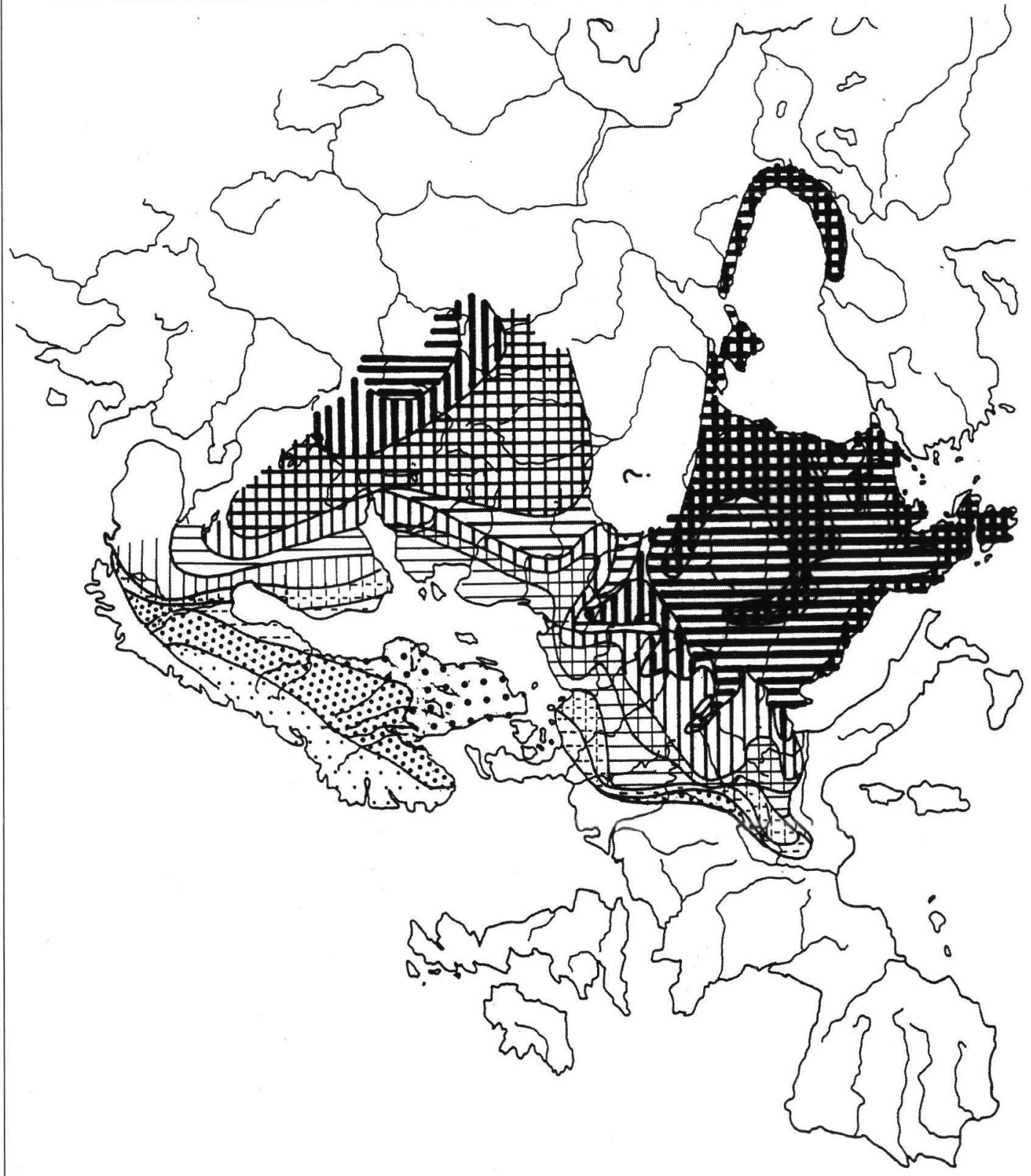




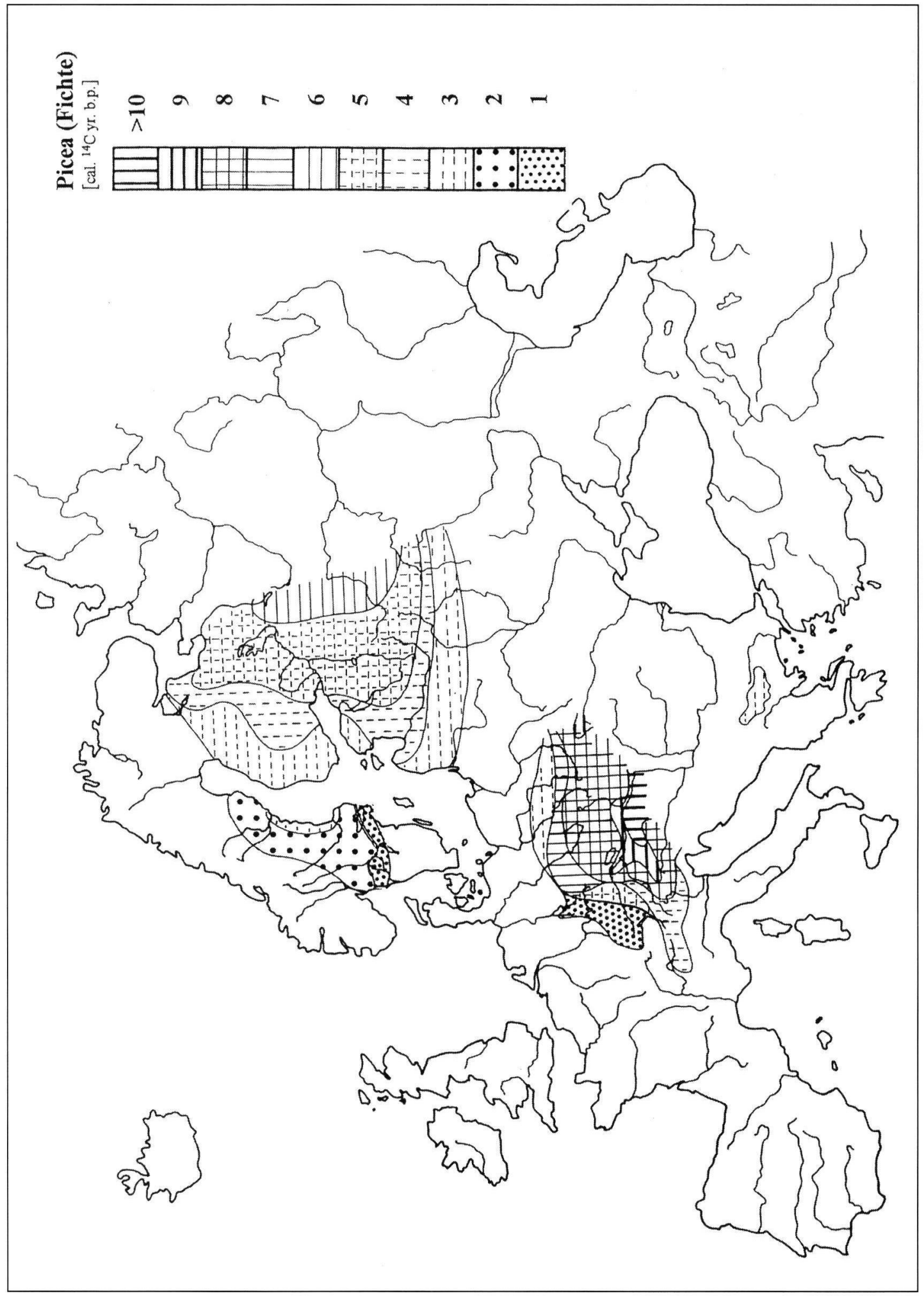




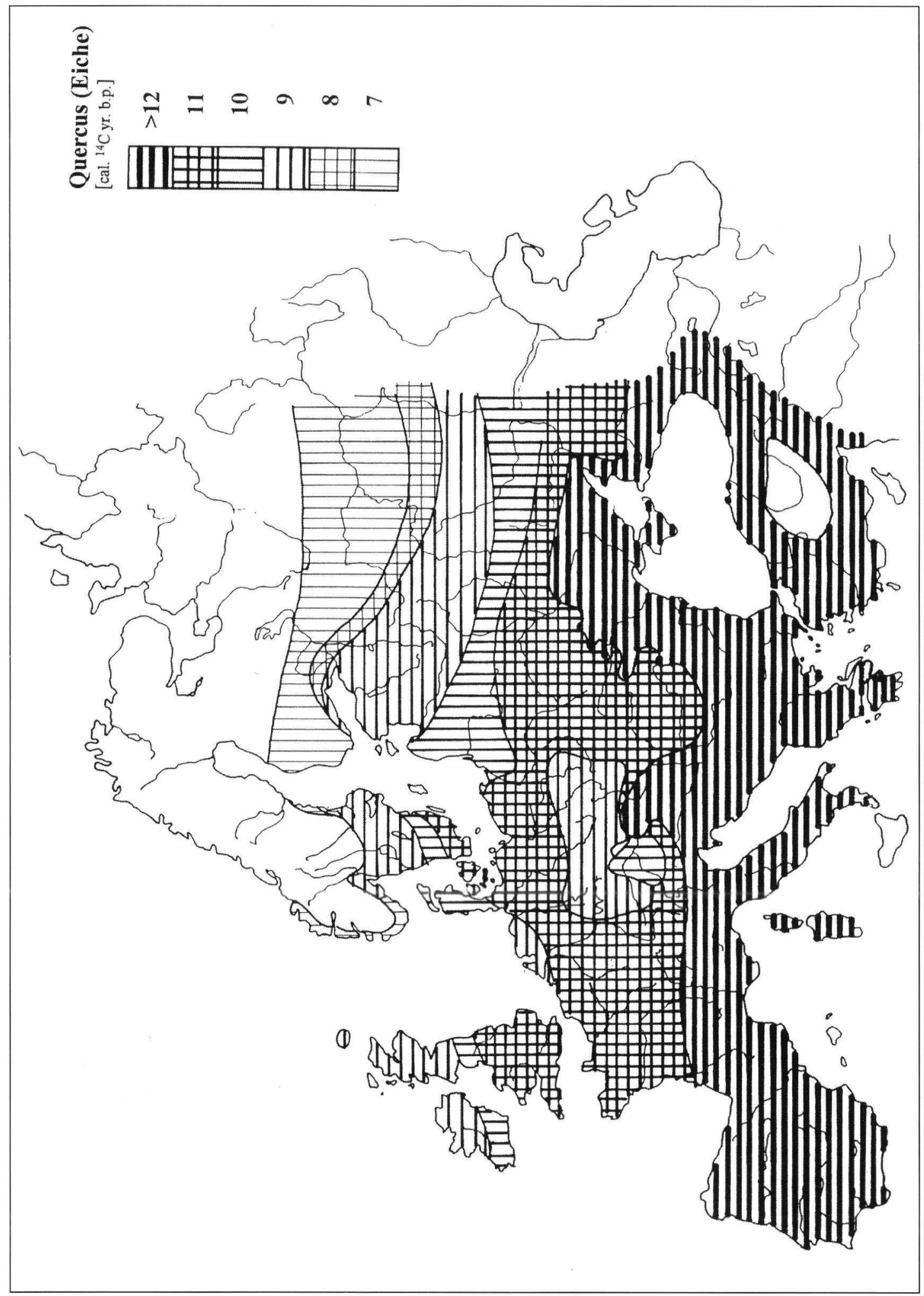


herrschenden Steppen vereinzelt oder in lichten Hainen auf (Abb. 3). Ab 12 '000 cal. ${ }^{14} \mathrm{C}$ yr. b. p. läßt sich eine Einwanderung von Quercus in einer typischen Süd-Nord-Bewegung nach Mittel- und Nordeuropa erkennen, nur in Süddeutschland, der Slowakei, der Tschechei und Südpolen tritt eine zeitliche Verzögerung von 2000 Jahren in der Einwanderung der 1.Spuren von Quercus auf. 9'000 cal. 14C yr. b.p. hat die Eiche die Grenzen des Kontinentes mit England und Irland erreicht und breitet sich in den nächsten 2000 cal. Jahren nur noch in Skandinavien und Rußland weiter nach Norden aus. Mit dem Höhepunkt des Wärmeoptimums des Atlantikums um $7000 \mathrm{cal} .{ }^{14} \mathrm{C}$ yr. b.p. hört auch diese nach Norden gerichtete Einwanderung der 1.Spuren von Quercus auf. Vom Menschen ein sehr geschätzter Baum als Nutz- und Bauholz, für die Eichelmast, für die Gewinnung von Gerberlohe und im Niederwaldbetrieb verwendet, läßt sich allerdings der menschliche Einfluß beim Rückgang der Arealgrenzen in dieser Form der Kartenpräsentation nicht fassen, da die Eiche vor dem Eingriff des Menschen bereits mit ihren ersten pollenanalytischen Spuren den Kontinentrand erreicht hatte.

Betrachtet man die Massenausbreitung von Quercus in Abb. 4, so zeichnet sich eine Massenausbreitung in Südeuropa vor 10000 cal. ${ }^{14} \mathrm{C}$ yr. b. p. ab. In den nächsten 1000 cal. Jahren setzt sich diese Massenausbreitung im nördlichen Alpenvorland fort und, geht dann in den darauffolgenden 1000 cal. Jahren fingerförmig nach Norden und Nordosten weiter. Letzte Hinweise auf die Massenausbreitung der Eiche lassen sich in der Zeitscheibe $5000 \mathrm{cal} .{ }^{14} \mathrm{C}$ yr. b. p. in Irland, Dänemark und Südschweden fassen.

\subsection{Vergleichende Betrachtung der Ergebnisse der Einwanderung der 1. Spuren und der Massenausbreitung}

\subsubsection{Ausbreitungsdauer von den 1. Spuren bis zur Massenausbreitung}

Die zeitliche Dauer, die zwischen dem Auftreten der 1. Spuren einer Baumgattung am Standort und ihrer Massenausbreitung liegt, kann bei ein und derselben Baumgattung an den einzelnen Standorten unterschiedlich sein. Abb. 5 versucht dies zusammenfassend über alle untersuchten Standorte darzustellen. Hierbei gibt die Ordinate die Zeitdauer wieder, aufgetragen in Abschnitten von 500 cal. Jahren, die die Baumgattung benötigt, um von den 1. Spuren bis zur Massenausbreitung zu gelangen. Auf der Abszisse sind in Prozent die Standorte aufgetragen, an denen die Baumgattung diese Zeitdauer benötigt. Bei Quercus zum Beispiel dauert der Schritt von dem Auftreten der 1. Spuren bis zur Massenausbreitung an 30\% der Standorte weniger als 500 cal. Jahre, an
15\% kommt es nach 500 cal. Jahren zum Auftreten einer Massenausbreitung nach der Einwanderung der 1.Spuren, an 20\% der Standorte liegt die Zeitdauer zwischen 1000 und $1500 \mathrm{cal}$. Jahren und bei 10\% der Standorte braucht dieser Schritt 1500 bis 2000 cal. Jahre. An den restlichen Standorten, an denen Quercus zur Massenausbreitung kommt, dauert der Schritt von den 1. Spuren zur Massenausbreitung zwischen 2000 und 6000 cal. Jahre.

Aus Abb. 5 läßt sich erkennen, daß bei der Baumgattung Quercus der Schritt von den 1.Spuren bis zur Massenausbreitung an den meisten Standorten in den ersten 500 Jahren stattfindet und nur an wenigen Standorten dieser Schritt länger als 2000 Jahre dauert. Dagegen ist die Zeitdauer zwischen dem Auftreten der 1. Spuren und der Massenausbreitung bei Picea an den Standorten etwa gleichverteilt in den ersten 2000 Jahren.

\subsubsection{Ausbreitungsdauer von den 1. Spuren bis zur Massenausbreitung in Abhängigkeit von den bereits am Standort vorkommenden Konkurrenten}

Die Zeitdauer, die zwischen dem Auftreten der 1. Spuren und der Massenausbreitung einer Baumgattung am Standort vergeht, kann von zahlreichen Faktoren abhängen. Neben für die Baumgattung ungünstigen Standortbedingungen, die eine Massenausbreitung nicht ermöglichen, können es auch die bereits am Standort vorkommenden Konkurrenten sein, die als Hemmnisse auftreten. Aus den Pollendiagrammen lassen sich die ökologischen Standortbedingungen, wenn überhaupt, dann nur äußerst zeitaufwendig und schwierig rekonstruieren. Daher wird im vorliegenden Fall nur die Zeitdauer, die eine Baumgattung braucht, um am Standort von den 1. Spuren bis zur Massenausbreitung zu kommen verglichen mit der Zeitdauer, die die anderen am Standort vorkommenden Baumgattungen für diesen Schritt benötigen. Hierfür wurden insgesamt 13 Baumgattungen (Abies, Acer, Alnus, Carpinus, Corylus, Fagus, Fraxinus, Picea, Populus, Quercus, Taxus, Tilia, Ulmus) gemeinsam betrachtet (GLIEMEROTH, 1995).

Hinter Abb. 6 verbirgt sich somit eine Aussage über die Durchsetzungsfähigkeit der Baumgattung in Form der Zeitdauer des Auftretens der 1. Spuren bis zur Massenausbreitung gegenüber den anderen Baumgattungen am Standort. Diese Betrachtung ist allerdings nicht mit der rezenten Konkurrenz- und Durchsetzungsfähigkeit einer Baumgattung am Standort gegenüber anderen Baumgattungen zu vergleichen, da die Baumgattungen zu unterschiedlichen Zeiten eingewandert sind. Wenn somit Quercus an den meisten Standorten, an denen sie zur Massenausbreitung gelangt, die Baumgattung ist, die im Vergleich mit den anderen am Standort vorkom- 


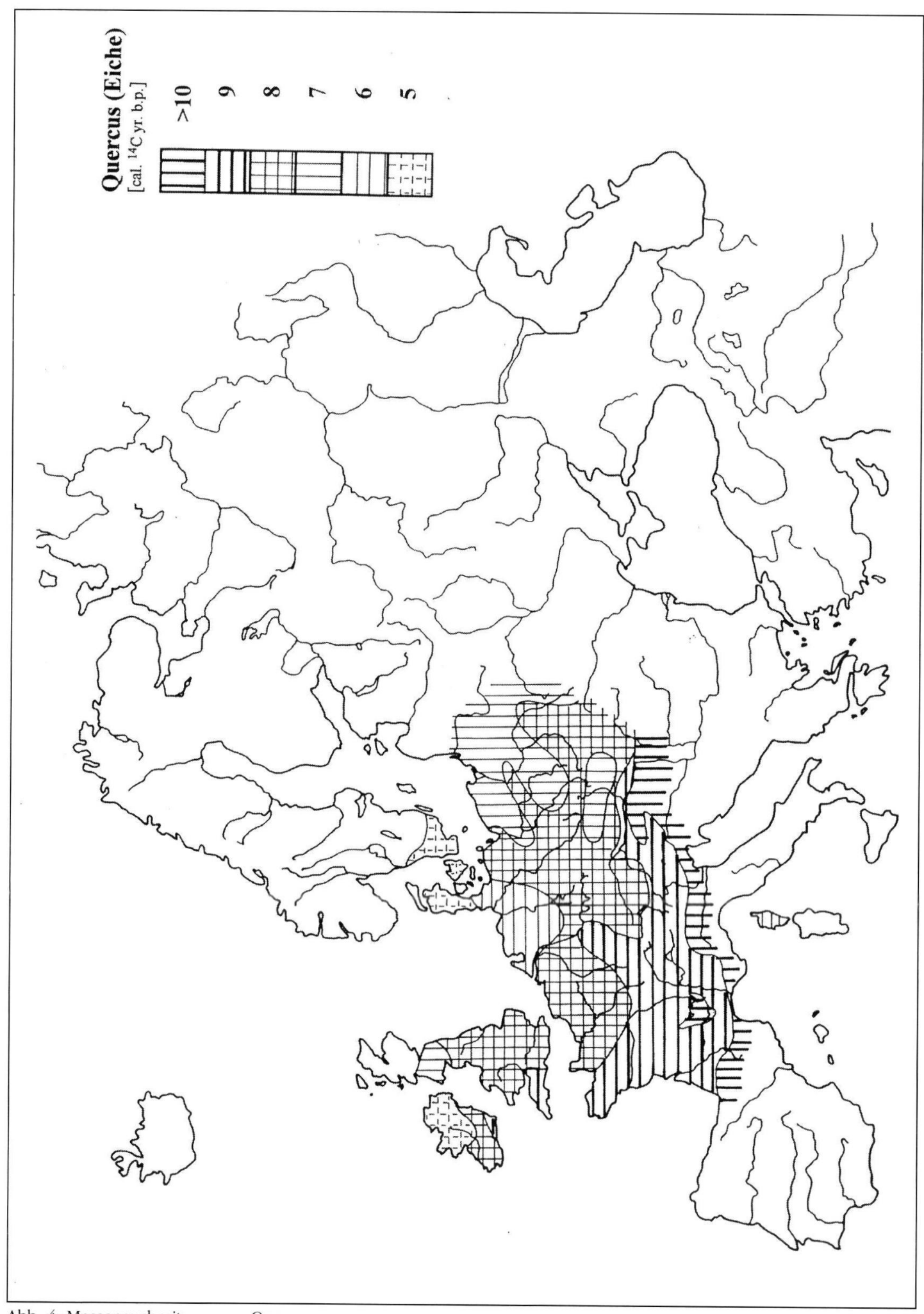




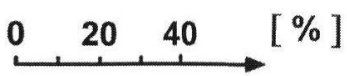

[ cal. ka ]

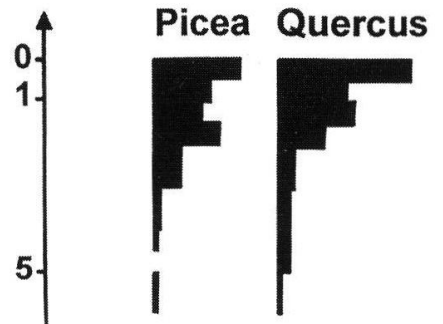

10.

Abb. 5: Zeitdauer zwischen dem Auftreten der 1. Spuren einer Baumgattung und ihrer Massenausbreitung, wobei die Anzahl aller Standorte an denen die Baumgattung zur Massenausbreitung gelangt, gleich $100 \%$ gesetzt wurde.

Fig. 5: Timespan between the appearance of first traces and mass spreading of tree taxa (100\% is the total amount of localities were the tree taxa comes to a mass spreading).

menden Baumgattungen hierfür die kürzeste Zeitdauer braucht, zeigt dies, daß Quercus zu dem Zeitpunkt, an dem sie an den Standorten einwanderte, günstige Bedingungen für eine Massenausbreitung vorgefunden hat, gegenüber den Bedingungen, die die anderen Baumgattungen bei ihrer Einwanderung für diesen Schritt am Standort vorfanden.

Quercus ist eine Baumgattung, die an vielen Standorten gegenüber den anderen vorkommenden Baumgattungen die kürzeste Zeit für den Schritt von den 1. Spuren bis zur Massenausbreitung brauchte. Bei Picea dauert der Schritt von den 1. Spuren bis zur Massenausbreitung an den meisten Standorten länger als bei zumindest einem Teil der vorkommenden Konkurrenten.

Es fällt auf, daß Quercus, die recht frühzeitig mit ihrer Einwanderungsbewegung aus den eiszeitlichen Refugien nach Europa beginnt und als 1. Spuren den Kontinent schnell besiedelt, auch für den Schritt von den 1.Spuren bis zur Massenausbreitung eine kürzere Zeit braucht als alle nachher einwandernden Konkurrenten. Picea scheint trotz ihrer späten Einwanderung an vielen Standorten Mitteleuropas sowohl positiv, wie negativ von den bereits am Standort vorkommenden Konkurrenten bei der Dauer des Schrittes von den 1. Spuren zur Massenausbreitung beeinflußt zu werden. Die Dauer für den Schritt von den 1.Spuren bis zur Massenausbreitung liegt daher im Vergleich mit den anderen am Standort vorkommenden Konkurrenten eher im Bereich der mittleren Zeitdauer.

\subsubsection{Anfang und Ende der Ausbreitung der 1. Spuren und der Massenausbreitung}

Der Nachweis des Beginns der Wanderbewegung der 1. Spuren einer Baumgattung aus ihren eiszeitlichen Refugien ist äußerst schwer zu fassen, da die Dichte der Pollendiagramme (Gliemeroth, 1995) in diesen Gebieten nicht sehr groß ist. Die Grenzziehung zur ersten, hier untersuchten Zeitscheibe einer Baumgattung ist daher etwas willkürlich und läßt nur die Aussage zu, daß die Baumgattung bis zu diesem Zeitpunkt sich in dem markierten Gebiet ausgebreitet hat, nicht aber, welche Zeit der Schritt bis zu dieser Grenze gebraucht hat und welche Wege dafür eingeschlagen wurden.

Trotzdem unterscheidet sich der Zeitpunkt dieser ersten Grenzziehung bei Picea und Quercus, so daß man eine zeitliche Einordnung des Beginns der Wanderbewegungen der 1 . Spuren vornehmen kann. Besonders früh läßt sich der Beginn der Einwanderungsbewegung ab $14000 \mathrm{cal} .{ }^{14} \mathrm{C}$ yr. b. p. bei Picea fassen. Ab 12000 cal. ${ }^{14} \mathrm{C}$ yr. b. p. wird bei

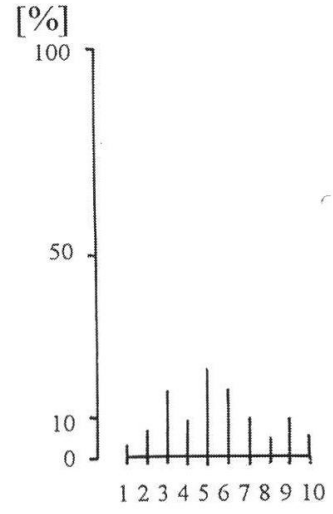

Picea

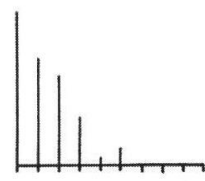

12345678910

Quercus
Abb. 6: Zeitdauer des Schrittes von den 1. Spuren bis zur Massenausbreitung (Abszisse) im Vergleich zu der Zeitdauer dieses Schrittes der anderen am Standort vorkommenden Konkurrenten. Rangfolge der Zeitdauer von 1 bis 10, im Vergleich zu den anderen Baumgattungen, angegeben in Prozent der Standorte, an denen die Baumgattung diese Rangstufe aufweist.

Fig. 6: Timespan between first traces and mass spreading in comparison to the timespan other tree taxa needs for this step at the same locality. Ranking of timespan from 1 to 10 given in percentage values of localities were the tree taxa shows this ranking. 
Quercus der Beginn der Einwanderung deutlich. Daß der Beginn der Ausbreitung der 1. Spuren einer Baumgattung aus dem eiszeitlichen Refugium besonders bei den früh einwandernden Baumgattungen primär mit einer Klimaverbesserung zusammenhängt, ist selbstverständlich. Der Beginn der Einwanderung bei der einzelnen Baumgattung wird allerdings, neben ihren zum Teil höheren klimatischen Ansprüchen, auch von Lage und Ausstattung der Refugien, von der physisch-geographischen Ausstattung der Wanderwege und den ökologischen Verhältnissen beeinflußt, die diese Baumgattung am Standort vorfand. So sind das Standortklima und die Bodenentwicklung Faktoren, die von den vor der betreffenden Baumgattung eingewanderten Konkurrenten bereits zugunsten der Baumgattung verändert werden können.

Die Zeitdauer, die zwischen dem Anfang und dem Ende der Erfassung der Wanderbewegung der 1. Spuren bei den einzelnen Baumgattungen liegt, ist äußerst unterschiedlich. Ganz besonders lange läßt sich die Veränderung der Arealgrenzen der 1. Spuren bei Picea fassen $\left(14^{\prime} 000 \mathrm{cal} .{ }^{14} \mathrm{C}\right.$ yr. b.p. bis heute). Nur einen relativ kurzen Zeitraum von 3000 Jahren umfaßt dagegen die Wanderbewegung der 1. Spuren bei Quercus.

Auf der Suche nach Gründen für das Ende der Ausbreitung der 1. Spuren einer Baumgattung wurde versucht, möglichst viele Faktoren zu erfassen. So macht ein Blick auf die Ausbreitungskarten der 1. Spuren von Quercus einen Grund für das Ende der Ausbreitung der 1.Spuren an Teilen der Arealgrenze deutlich. Besonders in den westlichen Bereichen ihrer Ausdehnung erreicht diese Baumgattung den Rand des Kontinents, so daß eine weitere Ausdehnung des Areals in diese Richtung nicht mehr gefaßt werden kann. Allerdings stößt die Wanderbewegung der 1. Spuren bei Quercus an der Nordgrenze des Areals nicht an die Kontinentgrenze, so daß für die Ausbildung dieser Grenze klimatische Faktoren von Bedeutung sein könnten. Für einen Einfluß des Klimas auf die Ausbildung dieser Nordgrenze spricht das Erreichen der Nordgrenze bei Quercus während der klimatisch günstigen Verhältnisse des holozänen Wärmeoptimums im Atlantikum 7'000 cal. ${ }^{14} \mathrm{C}$ yr. b. p.. Picea scheint im Moment noch in Ausbreitung begriffen zu sein, die sich unabhängig vom zunehmenden menschlichen Einfluß auch in den letzten 2000 cal. Jahren noch fassen läßt.

Auch beim Beginn und Ende der Massenausbreitung der hier untersuchten Baumgattungen soll die Frage nach einem möglichen Einfluß des Klimas auf diese Prozesse erörtert werden. Allerdings ist bei einem solch komplexen Vorgang wie ihn der Beginn der Massenausbreitung darstellt einsichtig, daß neben dem Faktor Klimaerwärmung auch ökosysteminterne Faktoren wie das Herausbilden eines Bestandes- klimas, die fortschreitende Bodenentwicklung etc. eine ganz entscheidende Rolle spielen.

Bei Quercus und Picea beginnt die Massenausbreitung in einzelnen Gebieten bereits um $10^{\prime} 000 \mathrm{cal} .{ }^{14} \mathrm{C}$ yr. b. p., was mit dem Ende des Spätglazials und dem Übergang zum Postglazial zusammenfällt und steht daher sicher mit einer Klimaverbesserung und der einsetzenden Bodenentwicklung in Zusammenhang.

Das Ende der Neubesiedlung von Gebieten, in denen die Baumgattung Quercus zur Massenausbreitung gelangt, liegt in der Zeitscheibe $5^{\prime} 000 \mathrm{cal} .{ }^{14} \mathrm{C}$ yr. b. p.. Ob das Ende der Massenausbreitung ein Hinweis auf die Klimaverschlechterung nach dem holozänen Wärmeoptimums im Atlantikum $\left(7^{\prime} 000\right.$ cal. ${ }^{14} \mathrm{C}$ yr. b. p.) ist, erscheint gerade für diese Baumgattung wegen ihres frühen Beginns der Massenausbreitung in der klimatisch noch recht ungünstigen Zeitscheibe $10^{\prime} 000$ v.h. unwahrscheinlich. Bei Picea könnte das Ende der Massenausbreitung $11^{\prime} 000 \mathrm{cal} .{ }^{14} \mathrm{C}$ yr. b. p. mit ihrer Nutzung und damit ihrer Beeinflussung durch den Menschen zusammenhängen.

\section{Diskussion}

Die hier erstellten Einwanderungskarten der 1. Spuren der Baumgattungen sowie ihrer Massenausbreitung sind in ihrer Darstellungsform der „Überlagerungskarten" sehr verschieden von den bisher erstellten Isopollenkarten (BERNABO \& WEBB, 1977; Huntley \& Birks, 1983; RalsKa-JaSIEWICZOWa, 1983). Als erstes mußten für die einzelnen Baumgattungen die Grenzwerte festgelegt werden, ab welchen man von den 1. Spuren und der Massenausbreitung bei der Baumgattung sprechen kann. Diese Prozentwerte in den Pollendiagrammen wurden nach der Pollenproduktivität, der Transportfähigkeit des Pollens und der Pollenkonservierung vorgenommen. Trotz der großen Sorgfalt, mit der bei der Festlegung der Pollenprozentwerte vorgegangen wurde, muß darauf hingewiesen werden, daß durch diesen methodischen Ansatz eine gewisse Fehlerbreite bei der Grenzziehung in den „Überlagerungskarten“ auftreten kann, die aber durch die Vorteile bei der Beantwortung vegetationskundlicher und ökologischer Fragen durch diese Karten akzeptiert wurde.

Die vorliegenden Karten ermöglichen es, sich einen relativ schnellen Überblick über die Einwanderungsrichtung, die benutzten Einwanderungsbahnen, die maximal eingenommenen Arealgrenzen, die Einwanderungszeiten und die Einwanderungsgeschwindigkeit zu machen. Hierin sind sie den Isopollenkarten (HuntLeY \& Birks, 1983) überlegen, die zur Beantwortung dieser Fragestellungen ungeeignet erscheinen. Allerdings sind die vorliegenden „Überlagerungskarten" nicht in der Lage, nach der Einwanderung der Baumgattungen ihre eventuelle 
Ausdünnung oder sogar Auswanderung aus einem Gebiet darzustellen, was besonders bezüglich des Rückzugs der Baumgattungen nach ihrer maximalen Ausbreitung äußerst interessant wäre. Es ist deshalb geplant, mit diesem Datenmaterial sowie unter Verwendung der absoluten Datierung, ebenfalls „Isopollenkarten“ zu erstellen. Für die Einwanderungsrichtung ist die Lage der eiszeitlichen Refugien von großer Bedeutung. Unter dem Begriff des „eiszeitlichen Refugiums" werden in diesem Zusammenhang Gebiete verstanden, von denen aus die erste größere Wanderbewegung der 1 . Spuren der einzelnen Baumgattungen zu fassen ist. Für Quercus konnten als eiszeitliche Refugien die nördliche Umrandung des Schwarzen Meeres, die Türkei, die Balkan-Halbinsel, Italien, der Ostrand der Alpen, Süd-Frankreich und die Iberische Halbinsel erfaßt werden, für Picea die nördliche Umrandung des Schwarzen Meeres, die Balkan-Halbinsel sowie Mittelrußland. Die Pollenprozentwerte der Baumgattungen können in den eiszeitlichen Refugien zum Teil recht hoch sein. Meistens sind in den einzelnen eiszeitlichen Refugien mehrere Baumgattungen vertreten gewesen, die mit ihrer Einwanderungsbewegung aus diesen Refugien oft zu unterschiedlichen Zeiten beginnen und auch verschiedene Einwanderungsgeschwindigkeiten aufweisen (GLIEMEROTH, 1995).

Viele der Refugien lagen also in Süd- und Südosteuropa. Die Gründe dafür, daß sich in einem Gebiet ein eiszeitliches Refugium ausbilden konnte, sind im einzelnen noch unklar, doch kann man sicher davon ausgehen, daß die klimatischen Bedingungen, die sowohl großräumig wie lokalklimatisch herrschten, von großer Bedeutung waren. Aber auch ein möglichst vielfältiges Relief, zur Ausbildung einer großen Anzahl unterschiedlicher Habitate, ist sicher von Vorteil gewesen. So finden sich die meisten Refugialräume in gebirgigen Gebieten, die durch die Ausbildung unterschiedlicher Höhenstufen, unterschiedlicher Expositionslagen etc. mannigfaltige ökologische Nischen zum Überdauern boten. Daß allerdings auch der Zufall beim Überleben einer kleinen, isolierten Population in einem Refugium eine Rolle spielen kann, zeigte RatcLiffe (1960).

In einigen der Refugien treten zahlreiche Holzpflanzengattungen auf, andere Refugien wurden nur von einzelnen Baumgattungen benutzt. Dies hängt sicher mit den ökologischen Ansprüchen der Baumgattung und ihren „Auswanderungswegen“ zu Beginn der Letzten Eiszeit aus Europa zusammen. Trotzdem wirft dieser Punkt erneut Licht auf eines der größten Probleme, das bei der Erstellung der Karten auftrat: Die Pollenanalyse ist, bis auf wenige Ausnahmen, nicht in der Lage, bei den hier untersuchten Baumgattungen eine Bestimmung des Pollens unterhalb des Gattungsniveaus durchzuführen. Die einzelnen Arten einer Gattung sind zum Teil je- doch in ihren ökologischen Ansprüchen recht unterschiedlich und können daher die in diesem Zusammenhang diskutierten unterschiedlichsten Refugialräume besiedeln.

Auch die Einwanderungsgeschwindigkeit ist bei den einzelnen Baumgattungen recht unterschiedlich. Während Picea mit häufigen Stops in ihrer 14000 cal.Jahren dauernden Einwanderungsgeschichte zu kämpfen hat, wandert Quercus in recht großen Schritten ein. Für die Größe dieser Schritte, das heißt die sogenannte Einwanderungsrate, ist es schwierig, eine Erklärung zu finden, da die Bäume zur Geschlechtsreife kommen müssen, bevor sie neue Früchte oder Samen bilden können, die den nächsten Sprung nach vorne einleiten können.

Vergleicht man die Ausbreitungsgeschwindigkeiten der Baumgattungen zwischen Europa und Nordamerika (DAvIs, 1976), so zeigt sich, daß sich in Nordamerika keine großen Einwanderungssprünge erkennen lassen. Mit ein Grund hierfür könnte sein, daß die Flüsse Nordamerikas in West-Ost oder NordSüd-Richtung verlaufen, so daß ein Transport der Früchte durch sie nicht bei der Ausbreitung einer Baumgattung nach Norden ins Gewicht fällt.

Neben diesen die Ausbreitung zum Teil begünstigenden Süd-Nord verlaufenden Flußsystemen stellen sich in Europa jedoch die West-Ost verlaufenden Gebirge als große Barrieren einer Ausbreitung der Baumgattungen in den Weg. Die meisten Baumgattungen umgehen auf bestimmten, günstigen Einwanderungsbahnen die Gebirgszüge. Für die Alpen sind dies im Osten das Donautal und im Westen die Burgundische Pforte. Allerdings bieten gerade in den Alpen die großen Transversaltäler die Möglichkeit zur Einwanderung der Baumgattungen (BurGA, 1980; ZOLLER, 1987).

Aber auch die im Norden gelegenen Meeresbarrieren des Ärmelkanals und der Irischen See haben sicher die Nordwest-Ausdehnung einiger Baumgattungen behindert (GoDwIN, 1975), während im Osten die Ostsee eine Barriere für die Einwanderung der Baumgattungen darstellte, deren beeindruckendstes Beispiel die Einwanderung von Picea aus dem Osten nach Südschweden ist. Neben der Einwanderungsroute über die Ålands-Inseln wanderte Picea von Osten um den Bottnischen Meerbusen nach Norden, von Finnland über Nord- nach Südschweden. Im Westen scheinen dagegen die dänischen Inseln eine recht ungehinderte Einwanderung von Picea nach Südschweden ermöglicht zu haben.

Eine genauere Analyse der Zeitdauer vom Auftreten der 1. Spuren bis zur Massenausbreitung bei den Baumgattungen macht deutlich, daß die Zeitdauer, die die einzelnen Baumgattungen für diesen Schritt brauchen, spezifisch für die Baumgattung ist. Dies bestätigte sich auch bei der Untersuchung weiterer 
Baumgattungen wie Abies, Alnus, Carpinus, Corylus, Fagus, Fraxinus, Tilia und Ulmus (GliemeROTH, 1995). Eine weitere Untersuchung konnte Aufschluß darüber geben, inwieweit sich die Dauer des Schrittes von den ersten Spuren bis zur Massenausbreitung bei den Baumgattungen eines Standortes voneinander unterscheidet. Dadurch läßt sich zeigen, daß Quercus an vielen Standorten gegenüber den anderen, gemeinsam vorkommenden Baumgattungen die kürzeste Zeitspanne zwischen dem Auftreten der 1. Spuren und der Massenausbreitung aufweist. Die Zeitdauer, die der Schritt von den 1. Spuren bis zur Massenausbreitung bei den Baumgattungen benötigt, wird, neben anderen Faktoren, durch die bei der Einwanderung der Baumgattung bereits am Standort vorkommenden Konkurrenten, die durch sie in Gang gesetzte Entwicklung eines Bestandesklimas und die Bodenentwicklung beeinflußt.

Die Erstellung der vorliegenden „Überlagerungskarten" zur Einwanderungsgeschichte der 1. Spuren der hier untersuchten Baumgattungen sowie ihrer Massenausbreitung lassen sehr deutlich baumgattungsspezifische Phänomene erkennen, die sich in dieser Form nicht aus „Isopollenkarten" erfassen lassen und machen durch die Fülle ihrer Ergebnisse die Komplexizität der Vegetationsentwicklung im Holozän deutlich.

Für die Einwanderungsgeschichte scheinen geologische und biologische Prozesse, wie die eiszeitliche Lage der Refugien, die eingeschlagenen Wanderbahnen, die Möglichkeit des Transportes der Früchte und Samen durch Wind, Wasser oder Tiere, die Einwanderungszeit, die Konkurrenzstärke der Baumgattungen und die in den sich ausbildenden Ökosystemen vorhandenen Regelmechanismen, wichtiger gewesen zu sein, als die mit anderen Mitteln sonst klar nachweisbaren Klimaschwankungen. Dieses individuelle Verhalten der Baumgattungen ist nicht begrenzt auf die Einwanderungsbewegungen der hier untersuchten letzten $14^{\prime} 000 \mathrm{cal} .{ }^{14} \mathrm{C}$ yr. b. p., sondern trat bereits in den vorangegangenen Interglazialen auf (FrenzEL, 1968; WATTS, 1973; DAvIS, 1976; West, 1980). Hieraus entstand die Vorstellung, daß die uns heute entgegentretenden Pflanzengesellschaften keine lange gemeinsame Geschichte haben, sondern nur mehr oder weniger zufällige $\mathrm{Zu}$ sammenschlüsse der verschiedenen Pflanzen unter den heutigen Klimabedingungen sind (WEST, 1964). Diese Vorstellung, daß die heutigen Pflanzengesellschaften und Ökosysteme keine starren Gebilde sind, sondern in Abhängigkeit von äußeren und inneren Faktoren zusammengefügte und sich im Verlauf der Zeit verändernde Gemeinschaften, zeigt die Fähigkeit auf, mit der heutige Ökosysteme prinzipiell in der Lage sind, auf unterschiedlichste Veränderungen ihrer Umgebung zu reagieren (FrENZEL \&
GLiemeroth, 1995). Durch den zunehmenden Einfluß des Menschen nicht nur auf die Ökosysteme selbst, sondern auch durch Städte- und Straßenbau sind jedoch die Reaktionsfähigkeiten heute eingeschränkt.

\section{Schriftenverzeichnis}

AARIO, R. (1965): Die Fichtenverhäufung im Lichte von ${ }^{14} \mathrm{C}$-Bestim mungen und die Altersverhältnisse der Finnischen Pollenzonen. - C.R.Soc.Geol.Fin., 37: 215-231; Helsinki.

Aartholathi, T. (1966): Über die Einwanderung und die Verhäu fung der Fichte in Finnland. - Annls.bot.fenn., 3: 368-379; Helsinki.

- (1967): Zur rationellen Tilia-Pollengrenze $\left(\mathrm{T}_{0}\right)$ in Finnland. Fennia, 97: 1-30; Helsinki.

BARD, E.; Fairbanks, R. G.; ARnold, M. \& Hamelin, B. (1993): ${ }^{230} \mathrm{TH} /{ }^{234} \mathrm{U}$ and ${ }^{1+} \mathrm{C}$ ages obtained by mass spectrometry on corals from Barbados (West Indies), Isabela (Galapagos) and Mururoa (French Polynesia). - In: BARD, E. \& BROECKER, W. S. (Hrsg.): The last deglaciation: Absolute and radiocarbon chronologies. NATO ASI Series, 12. -Springer, 103-110; Berlin.

Becker, B. \& Kromer, B. (1993): Tree-ring ${ }^{14} \mathrm{C}$-calibration at 10 '000 b.p. - In: BARD, E. \& BROECKER, W.S. (Hrsg.): The last deglaciation: Absolute and radiocarbon chronologies. NATO ASI Series, 12. - Springer, 3-11; Berlin.

BernaBo, J. C. \& WEBB, T. III (1977): Changing patterns in the Holocene pollen record of northeastern North America: a mapped summary. - Quat.Res., 8: 64-96; New York.

Birks, H. J. B.; WebB, T. III \& BerTi, A. A. (1975): Numerical analysis of surface samples from central Canada: a comparison of methods. - Rev. Palaeobotan. Palynol, 20: 133-169; Amsterdam.

Burga, C. A. (1980): Pollenanalytische Untersuchungen zur Vegetationsgeschichte des Schams und des San Bernhardino-Passgebietes (Graubünden). - Diss.Bot., 56: 1-165, Cramer, Stuttgart.

DAvIS, M. B. (1976): Pleistocene biogeography of temperate deciduous forests. - Geoscience and Man, 13: 13-26.

DONner, J. J. (1963): The zoning of the postglacial pollen diagrams in Finland and the main changes in the forest composition. Acta Bot.Fenn., 65: 2-40; Helsinki.

Firbas, F. (1949): Spät- und nacheiszeitliche Waldgeschichte Mitteleuropas nördlich der Alpen. Band 1.- 1-445; Fischer, Jena.

Frenzel, B. (1957): Beobachtungen zum Problem des Pollenfluges in einem Alpental. - Unveröff.Manuskript.

- (1960): Die Vegetations- und Landschaftszonen Nordeurasiens während der letzten Eiszeit und während der postglazialen Wärmezeit. - 1-453; Steiner, Wiesbaden.

- (1968): Grundzüge der pleistozänen Vegetationsgeschichte Nord-Eurasiens. - 1-326; Steiner, Wiesbaden.

- (1975): The distribution pattern of Holocene climate change in the Northern Hemisphere. - Proc.WMO/AMAP symp. on long-term climatic fluctzuations, 1975: 105-118; Norwich.

- (1992) ed.: Evaluation of land surfaces cleared from forests by prehistoric man in Early Neolithic times and the time of migrating German tribes. - Paläoklimaforschung, 8: 1-225; Fischer, Stuttgart

- (1993): Klimaschwankungen und der Vorgang der Ökosystemänderung. - In: Thews, G. \& Servatius, C. (Hrsg.): Ökosystemanalyse und Umweltforschung in Rheinland-Pfalz, Fischer, 11-26, Stuttgart.

- \& Gliemeroth, A. K. (1995): Zur Vegetationsgeschichte Mittel europas. - Angewandte Landschaftsökologie, 4: 15-50, Bonn.

Gliemeroth, A. K. (1995): Paläoökologische Untersuchungen über die letzten 22'000 Jahre in Europa: Vegetation, Biomasse und Einwanderungsgeschichte der wichtigsten Waldbäume. Paläoklimaforschung, 18, Fischer, 1-252, Stuttgart.

Godwin, H. (1975): The History of the British Flora. - University Press, 1-435, Cambridge.

Huntley, B. \& Birks, H.J.B. (1983): An atlas of past and present pollen maps for Europa: 0-13'000 years ago. - University Press, 1-854. Cambridge.

MoE, D. (1970): The postglacial immigration of Picea abies into Fennoscandia. - Bot.Notiser, 123: 61-66; Oslo. 
Moran, J. M. (1973): The late-glacial retreat of 'Arctic' air as suggested by onset of Picea decline. - Prof. Geogr., 25: 373-376.

RALSKA-JASIEWICZOWA, M. (1983): Isopollen maps for Poland: 0-11'000 years B.P.. - New Phytol., 94: 133-175.

Ratcliffe, D. A. (1960): The mountain flora of Lakeland. Proc.bot.soc.Br.Isl., 4: 1-25; London.

Rybnickova, E. \& Rybnicek, K. (1988): Isopollen maps of Picea abies, Fagus sylvatica and Abies alba in Czechoslovakia - their application and limitations. - In: LANG, G. \& SchNeIDER, C. (Hrsg.): Lake, mire and river environments during the last 15'000 years, Balkema, 51-66, Rotterdam.

Serebryanny, L. R. (1973): Postglacial migration rates of tree species in the northwestern regions of the USSR: palynology and radiocarbon dating. - In: KHotinsky, N.A. \& Koreneva, E.V. (eds.) Palynology: Holocene and marine palynology, Nauka, 51-78, Moskva.

SRODÓn, A. (1960): Pollen spectra from Spitsbergen. - Folia Quat., 3: 1-17; Warsawa.

Stutver, M.; Porter, S.C.; Mook, W.G.; Oeschger, H.; Davis, R.B.; MOORE, A. \& Kra, R.S. (1986) eds.: Calibration Issue. - Radiocarbon, 28.2B: 805-1030, Tuscon.

Stuiver, M.; Braziunas, T. F.; Becker, B. \& Kromer, B. (1991): Climatic, solar, oceanic and geomagnetic influences on Late-Glacial and Holocene atmospheric ${ }^{14} \mathrm{C} /{ }^{12} \mathrm{C}$ change. - Quat.Res., 35: 124; New York.

Stuiver, M.; Long, A.; Devine, J.M. \& Kra, R.S. (1993) eds.: Calibration 1993. - Radiocarbon, 35.1: 1-244; Tuscon.

SzAFER, W. (1935): The significance of isopollen lines for the investigation of the geographical distribution of trees in the postglacial period. - Bull. l'Acad. Sci. Polon., B1935: 235-239; Warsawa.

WATTS, W.A. (1973): Rates of change and stability in vegetation in the perspective of long periods of time. - In: BiRKS, H.J.B. \& WEST, R.G. (Hrsg.) Quaternary Plant Ecology, Blackwell, 134187, Oxford.

WEST, R. G. (1964); Inter-relations of ecology and quaternary palaeobotany. - J. Ecol., 52: 47-57.

- (1980): Pleistocene forest history in East Anglia. - New Phytol., 85: 571-622.

ZOLLer, H. (1987): Zur Geschichte der Vegetation im Spätglazial und Holozän der Schweiz. - Mitt.Naturforsch.Ges.Luzern Eiszeitforsch., 29: 123-149; Luzern.

Manuskript eingegangen am 23. 10. 1995 